\title{
Appearance of Nurses and Perceived Professionalism
}

\author{
Nikki L. Wills ${ }^{1}$, Brittany Wilson ${ }^{2}$, Eva B. Woodcock ${ }^{3}$, Samuel P. Abraham ${ }^{4} \&$ Deborah R. Gillum ${ }^{5}$ \\ ${ }^{1}$ Orthopedic/Neuro Surgery, Saint Joseph Regional Medical Center, Mishawaka, Indiana, USA \\ ${ }^{2}$ Labor \& Delivery, Saint Joseph Regional Medical Center, Mishawaka, Indiana, USA \\ ${ }^{3}$ Emergency Department, Goshen Hospital, Goshen, Indiana, USA \\ ${ }^{4}$ Nursing, Bethel College, Mishawaka, Indiana, USA \\ ${ }^{5}$ Nursing, Bethel College, 1001 Bethel Circle, Mishawaka, Indiana, USA \\ Correspondence: Samuel P. Abraham, Associate Professor of Nursing, Bethel College School of Nursing, 1001 \\ Bethel Circle, Mishawaka, Indiana, 46545, USA.
}

Received: August 31, 2018

doi:10.20849/ijsn.v3i3.466
Accepted: September 13, $2018 \quad$ Online Published: September 17, 2018

URL: https://doi.org/10.20849/ijsn.v3i3.466

\begin{abstract}
Background: After completing a literature review on the topic of appearance and professionalism a knowledge gap was identified, relating to how individuals perceive professionalism based on appearance. First impressions are how patients form opinions of their nurses. Professionalism is influenced by many variables, such as hair, make-up, uniform, behavior, and image. Purpose: The purpose of this study was to explore perceptions of a nurse's appearance and projected professionalism. Method: A total of 120 students volunteered to participate in the study. The research question was: "How do college students perceive the professional appearance of the nurse?" This was a quantitative, cross-sectional, non-experimental study with a descriptive design. A qualitative question was also asked to complement the quantitative data. The survey contained 3 demographic questions and 18 items based on the participant's perception of the perceived professionalism using the given images. The data collected were analyzed using descriptive statistics. Orem's theory of self-care was used to guide this study. Results: The results confirmed the complex nature of the nursing image. Participants perceived a nurse who took extra time to improve appearance to be professional, trustworthy and least lazy. A not so prepared nurse was perceived to lack confidence and also to be less compassionate.
\end{abstract}

Keywords: professionalism and appearance, nurse appearance and professionalism, professional nurses, nursing attire, self-care

\section{Introduction}

Professionalism is perceived in many ways. The traits that make a person professional are up for debate. Identifying how and why something is considered professional will help determine what characteristics a professional nurse should possess. The purpose of this study was to determine the perceived professionalism of the nurse based on appearance using Orem's theory of self-care as the guiding theoretical framework.

\subsection{Background}

Nursing is an iconic profession that has been around for thousands of years. Hartfield et al. (2013) stated, "Nurses have outperformed other professions in the Gallup Poll rating" (p. 108). Nurses are highly respected and trusted health care providers. Many variables exist that influence a person's perception of another individual. There is a disparity in how registered nurses portray themselves in comparison with how patients perceive them professionally. The appearance of the nurse and perceptions of professionalism are important questions that require exploration in this study. Porr et al. (2014) noted, "The public notion according to which the registered nurse $(\mathrm{RN})$ is deemed a professional health-care provider by virtue of his or her uniform has persisted throughout the generations especially during the first few moments of the nurse-patient encounter. Professionalism is primarily conveyed by the RN's appearance, with uniforms being the major factor" (p. 150).

Nurses wear uniforms in hospitals and at doctor's offices while maintaining expectations of professionalism through appearance and behavior when providing care to their patients. Nurses are in the unique role of being the most trusted people in healthcare and maintaining trust is imperative when interacting with various individuals and aggregates in a professional setting. Fogle and Reams (2014) claimed, "Professional dress was also found to 
impact confidence in the wearer's ability and knowledge" (p. 52). When the nurse takes time to present a professional appearance, the patient takes time to notice and associates that with confidence and competence.

Conducting this study on a college campus offered insight from students as to how they perceived a professional nurse based on appearance. The modern nurse must be educated and licensed in the state where they are practicing. By presenting a professional appearance, the nurse can leave a positive first impression. When patients perceive professionalism, they feel that their health care needs are in the hands of capable and well-educated healthcare professionals.

\subsection{Problem Statement}

Nursing is a professional career. Patients rely on nurses to help save lives every day. Therefore, the problem is that many people do not know what influences the perception of the nurses' professionalism. Patients form an impression of the nurse within the first 12 seconds (Splendore, Burns, \& Choby, 2016). How a nurse appears is a key factor in how the patient will judge the nurse's care ability (Splendore et al., 2016). Patients like to see a nurse who appears professional, well-groomed, neat, clean hair, and conservative makeup for females (Splendore et al., 2016). There is an association between the time the nurse spends on appearance and quality of professionalism perceived by patients and their families.

\subsection{Purpose Statement}

The purpose of this study was to determine the perceived professionalism of the nurse based on appearance.

\subsection{Research Question}

How do college students perceive the professional appearance of the nurse?

\subsection{Review of the Literature}

\subsubsection{Database Used}

The evidence-based practice articles were found using the college library, OneSearch search engine, and the Cumulative Index to Nursing and Allied Health Literature (CINAHL) database. The articles were located using keywords such as "professionalism and appearance," "nurse appearance and professionalism," "Professional nurses," and "nursing attire and professionalism."

\subsubsection{Professionalism}

Akhtar-Danesh et al. (2013) defined professionalism as "knowledge, specialization, intellectual, and responsibility" (p. 248). They also indicated that professionalism is expressed in "one's image, attire, and expression" (p. 248). Demonstrating professionalism increases the patient's perceived perception of the nurse being professional (Akhtar-Danesh et al., 2013). Nurses should want to be perceived as professional to be seen as competent by patients.

Ghadirian, Salsali, and Cheraghi (2014) identified professionalism as being related to culture. Working in healthcare a nurse interacts with people from different cultures. Culture competency is required for appropriate care. Nurses must be perceived as professionals in all cultures (Ghadirian et al., 2014). A nurse must be aware of how to be considered professional in other cultures.

Clavelle, Goodwin, and Tivis (2013) helped to demonstrate how a nurse's image portrays professionalism. A patient's perception of a nurse is based on image, appearance, and ability to identify the nurse. The public perceives nurses based on the idea of how a nurse should look. Professionalism is based on personal appearance and hygiene (Clavelle et al., 2013). Patients feel more comfortable with a nurse who is seen as a professional. Nurses should strive to look professional.

\subsubsection{Appearance}

Porr et al. (2014) used a quantitative survey to explore how appearance influenced perceived professionalism. Porr et al. surveyed 43 patients on a medical-surgical floor ranging in age from 19-86 years old. Each participant was shown different pictures of nurses and asked to evaluate the nurse's appearance using 10 traits (Porr et al., 2014). With the information gathered, the researchers were able to identify how the nurse conveyed professionalism. Data exhibited that professionalism was conveyed by the RN's appearance (Porr et al., 2014). Appearance influences the professional image. Nurses should want to be seen as professional.

Professionalism has contributed to the nurse's behavior, adherence to the Code of Ethics, and personal appearance (Porr et al., 2014). Patients assess the RN's professionalism within seconds of the first interaction (Porr et al., 2014). Professionalism, attitude, mood, and trustworthiness are all based on the outward appearance 
of the nurse. Patients' impression of the professional appearance of the nurse is contingent on how the nurse looks (Porr et al., 2014).

A negative impression of the nurse's appearance was shown to decrease the patient's confidence in their nurse (Porr et al., 2014). Appearance was demonstrated to directly correlate to the quality of care the nurse delivered. When patients did not feel confident in the nurse providing their care, the following was seen: an increase in suffering, a decrease in healing, and longer recovery times (Porr et al., 2014). A direct correlation between the nurse's appearance and quality of care delivered was noted.

Splendore et al. (2016) demonstrated the importance of the first impression. The study revealed how a person's first impression could influence how they perceived the nurse based on appearance. People do not get a second chance to improve the first impression. Nurses need to make good first impressions. Respect comes from professionalism in performance and appearance (Splendore et al., 2016). Nurses must be respected and trusted to allow for the most successful outcomes.

While (2013) claimed nursing is iconic and conveys important messages. Nurses have a positive image to uphold. The nursing field upholds the image of a professional through professionalism. A negative image of a nurse will damage the work the nurse does (While, 2013). Nurses must uphold a positive image to convey positivity. Nurses should be seen as positive members of the healthcare team.

Clavelle et al. (2013) gave insight on how a nurse should look. A nurse should be clean, neat in appearance, knowledgeable, and be able to perform duties. A lot of pressure is placed on the nurse's personal hygiene. Nurses must properly care for themselves (Clavelle et al., 2013). The bottom line is nurses need care too.

\subsubsection{Image}

Hartfield et al. (2013) presented the idea that a nurse's image is based on professionalism, confidence, trust, and therapeutic relationships. The professional image of a nurse creates a public image of the nursing field. In nursing, the image of the nurse makes an emotional connection with the patients (Hartfield et al., 2013). The image is how the public views nurses. Conveying a positive image of the nurse is important for how the public perceives the nursing field (Hartfield et al., 2013). Nurses should be seen as productive members of the care team.

Pullen and Alley (2015) helped define a professional image. An image includes how a person looks; how a person carries themselves; how a person thinks and acts; and how they communicate with others. A positive professional image increases the perceived credibility of the nurse (Pullen \& Alley, 2015). Nurses need to be viewed as credible.

Appearance can create a positive or negative impression of a nurse (Pullen \& Alley, 2015). A negative image is created when a nurse wears wrinkled, dirty uniforms, has poor grooming, is unclean, has unkempt hair, and has poor posture. A negative image of nurses decreases credibility to the patient (Pullen \& Alley, 2015). Nurses should create a positive image for patients.

Fogle and Reams (2014) defined what influences a positive image of a nurse. Patients are comfortable with a nurse that represents a professional image. Their study indicated that patients like a clean, well-groomed nurse. Patients did not like to see unkempt hair that was on the nurse's shoulders. The study revealed that a positive image of the nurse was perceived to be professional (Fogle \& Reams, 2014).

Wocial, Sego, Rager, Laubersheimer, and Everett (2014) further defined that a nurse should be well-groomed, clean, and wear conservative makeup to portray a positive professional image. A positive image creates positive impressions. The nurses should make a good lasting impression on their patients.

\subsubsection{Brand}

Wocial et al. (2014) defined nursing as a brand. Emotions are attached to brands. In nursing, patients should feel attached to their nurses. A nurse must be well-groomed, and clean to uphold a positive brand image (Wocial et al., 2014). The nurses should want to create a positive brand for themselves.

\subsubsection{Behavior}

Wocial et al. (2014) outlined what behavior is deemed professional. Nurses were seen to be more professional when the behavior was approachable, respectful, attentive, caring, reliable, and informative. Behaviors negatively or positively affected how a nurse is perceived professionally (Wocial et al., 2014). Positive behavior creates positive impressions. 
Akhtar-Danesh et al. (2013) warned a nurse could be perceived as unprofessional by spreading rumors, talking about people behind their backs, or participating in gossip. A professional nurse should not display behaviors that are deemed unprofessional. No nurse should be seen as unprofessional.

\subsubsection{Communication}

Swan and McGinley (2016) demonstrated that communication influences all aspects of care. When patients do not trust or believe in their nurse, successful health care outcomes decrease. Communication can be both verbal and nonverbal. Communication is vital when providing care to a patient. Nurses should strive for positive open communication between them and their patients to better-serve their patient's needs.

\subsubsection{Character}

Splendore et al. (2016) described how respect impacted care. Respect was conveyed through performance and appearance. Nurses demonstrated character through their personal appearance. A patient judges a nurse's character within the first 12 seconds of meeting. Respect is formulated based on the appearance of the nurse. The character is professionalism in action. Nurses should be seen as having a positive character.

\subsubsection{Summary of the Literature Review}

Professionalism is influenced by the way a nurse appears (Splendore et al., 2016). Many founding characteristics of a nurse are based on their appearance. Professionalism is based on being well-groomed and projecting a professional attitude (Wocial et al., 2014). A nurse should take time to improve their appearance and avoid the unprofessional behavior. Patients perceive a nurse's quality of care based on first impressions (Clavelle et al., 2013). Appearance is a paramount issue for nurses. Though the patients' perceptions were well documented, the college students' perceptions regarding a nurse's appearance were not found in the search; therefore, the need for this study.

\subsection{Theoretical Framework}

Orem's theory of self-care was used to guide this study. Orem's theory is based on the belief that a person needs to engage in activities that maintain a high quality of life, health, and well-being (Orem, Renpenning, \& Taylor, 2003). The theory is based on the idea that people who take time for themselves lead a higher quality of life and have an increased overall well-being. This theory indicates that individuals who take care of themselves will be more capable of helping others (Orem et al., 2003).

The basis of this theory was used when analyzing the appearance of the nurse and perceived professionalism. If nurses maintained themselves, they can better care for patients. Predicting that a nurse who spends time preparing their outward appearance for work, they will be perceived as more professional. The survey items used in the study also included pictures of a nurse who spent (1) very little or no time, (2) a little time, and (3) sufficient time on personal appearance.

\subsection{Definition of Terms}

\subsubsection{Conceptual Definition}

Self-Care is part of life that is necessary for health, human development, and well-being (Orem et al., 2003). It is learned through human interactions and communication. It includes deliberate and systematic action to meet a known need for care. Self-care agency is an acquired ability of a mature and maturing person to know own human functioning and development. Self-care demands consist of the summation of care measures necessary to meet all of an individual's known self-care requisites. Nursing agency refers to the developed capabilities of persons educated as nurses that allow them to act, to know, and to help patients meet their therapeutic self-care demands and to regulate the development or exercise of the patient's self-care agency (Orem et al., 2003).

\subsubsection{Operational Definition}

The appearance of the nurse was based on the neatness of hair, makeup, and what the uniform looked like. Professionalism was perceived as neat, competent, respectful person. College students were those who attended school after high school, ages 18 - 26 years.

\section{Methodology}

\subsection{Research Methods and Design}

This study was quantitative, non-experimental, with a descriptive design. It was survey-based. A qualitative question was also added to further expand the findings. A cross-sectional sampling method was used because it represented the population and helped provide an insight into how college students perceived professionalism in 
nurse's appearance. Before research was conducted, the College Institutional Review Board (IRB) approval was obtained.

\subsection{Population and Sampling Frame}

The target population for this study was college students. The sample size was 120 college students both male and female who volunteered for the study. The convenience sampling method was used.

\subsection{Geographic Location}

The survey occurred at a college in northern Indiana, USA, outside the college library from mid-morning through mid-afternoon. An area was set up outside the library for students to complete the survey either before or after they entered the library. Store-bought candy was offered as an incentive for students to participate in the survey.

\section{Data Analysis}

Data analysis occurred after all surveys were completed. Incomplete surveys were discarded. The frequency and percentage were calculated for the demographic questions and described using a table. To analyze the data, the mean was calculated for each of the 18 survey statements, and then the standard deviation for each mean was calculated. The demographic data were categorized at the nominal level of measurement, and the data for the 18 Likert-type statements were categorized at the ordinal level of measurement. The one qualitative item produced common themes. The quantitative data were entered into excel for analysis.

\section{Survey Instrument}

\subsection{New Survey Instrument}

After a thorough review of the literature, the survey instrument was created by the researchers. The Likert-type scale used for perception statements were strongly disagree (1), disagree (2), agree (3), and strongly agree (4). The responses were based on three pictures with varying amounts of time spent on appearance. There were 18 statements in the survey. There were also three demographic items at the beginning of the survey to obtain more information about the population. One qualitative question was used to collect data to complement the quantitative findings.

\subsection{Instrument Reliability and Validity}

This survey did not have established reliability as it was created for this study. However, it was peer-reviewed by two peers and two nursing professors to obtain face-validity. Care was taken to protect from bias.

\section{Informed Consent and Confidentiality}

Informed consent was used for this survey. The data were kept anonymous by excluding the participants' names and other identifying information. Keeping the consent separate from the survey's in different envelopes ensured confidentiality. The National Institutes of Health (NIH) certificates entitled "Protecting Human Research Participants" was completed by the researchers. Approval from the librarian was also obtained to use the lobby prior to collecting data.

All collected data for this research were submitted to the School of Nursing, to be stored electronically for three years. The School of Nursing staff scanned the data into the computer and stored it on discs. These discs will be locked in a storage cabinet in a locked storage room. No one other than the nursing administrators or the research coordinators will have access to the documents.

\section{Results}

The surveys were collected, and the results were tallied. The surveys were examined for completeness. The frequency and percentage of the demographic items were calculated. Mean, and the standard deviation was calculated for the survey responses. Common themes among the qualitative item were identified. The results indicated what college students found to be the professional appearance of the nurse. A higher mean value showed agreement in the opinion of what was professional, prepared, confident, trustworthy, compassionate, and lazy. The standard deviation was calculated for each item to verify variability of the data. Results of the quantitative study were compared to the literature. Orem's theory of self-care determined perceived professionalism through the appearance of the nurse.

\subsection{Demographics}

A total of 120 college students participated in this study with ages ranging from 18 to 26 years. Table 1 illustrates the descriptive statistics of the participants. The most common age range was 18-20 (61\%). There 
were more females $(59 \%)$ than males $(41 \%)$ surveyed. Almost all participants, $119(99 \%)$ had seen a nurse in uniform.

Table 1. Descriptive statistics for participant demographics

\begin{tabular}{lll}
\hline Variable & $f$ & $\%$ \\
\hline Age range & 73 & 61 \\
$18-20$ & 38 & 32 \\
$21-23$ & 9 & 7 \\
$24-26$ & & \\
Gender & 49 & 41 \\
$\quad$ Male & 71 & 59 \\
Female & & \\
Have you seen the nurses in their & & 99 \\
uniforms? & 119 & 1 \\
$\quad$ Yes & 1 & \\
No & &
\end{tabular}

\subsection{Response to Research Question}

The research question was: "How do college students perceive the professional appearance of the nurse?" This portion of the survey contained 18 items related to opinions of perceived professionalism using three pictures varying in attentiveness. The items explored how people perceive each picture using terms such as professionalism (Q1), preparedness (Q2), confidence (Q3), trustworthiness (Q4), compassion (Q5), and laziness (Q6). The responses corresponded with a number related to the Likert-type scale rated 1 as strongly disagree to 4 strongly agree. The mean values demonstrated the agreement in responses and opinions. A mean of 2.5 or higher indicated an agreement in opinion.

\subsection{Picture A: Sufficient Time Spent on Personal Appearance}

In Table 2, the statements had a mean from 1.87 to 3.25, and for Figure 1. Picture A, most of the participants agreed, displayed professionalism $(\mathrm{M}=3.25, \mathrm{SD}=0.57)$, and preparedness $(\mathrm{M}=3.25, \mathrm{SD}=0.57)$. Other statements ranked lower, including trustworthiness $(\mathrm{M}=2.83, \mathrm{SD}=0.70)$, confidence $(\mathrm{M}=2.75, \mathrm{SD}=0.77)$, and compassion $(\mathrm{M}=2.43$, SD 0.66). The lowest level of frequency was laziness $(\mathrm{M}=1.87, \mathrm{SD}=0.71)$. Picture $\mathrm{A}$ was seen to be professional and demonstrated preparedness while lacking in compassion. Picture A was seen to be the least lazy.

Table 2. The appearance of the nurse and perceived professionalism Picture A

\begin{tabular}{lcc}
\hline Variable & M & SD \\
\hline I feel picture A shows professionalism & 3.25 & 0.57 \\
I feel picture A shows preparedness & 3.25 & 0.57 \\
I feel picture A shows trustworthiness & 2.83 & 0.70 \\
I feel picture A shows confidence & 2.75 & 0.77 \\
I feel picture A shows compassion & 2.43 & 0.66 \\
I feel picture A shows laziness & 1.87 & 0.71 \\
\hline
\end{tabular}

Note. $(N=120)$. Student participants answered using a 4-point Likert-type scale, where 1 is the lowest agreement (strongly disagree), and 4 is the highest level of agreement (strongly agree). 


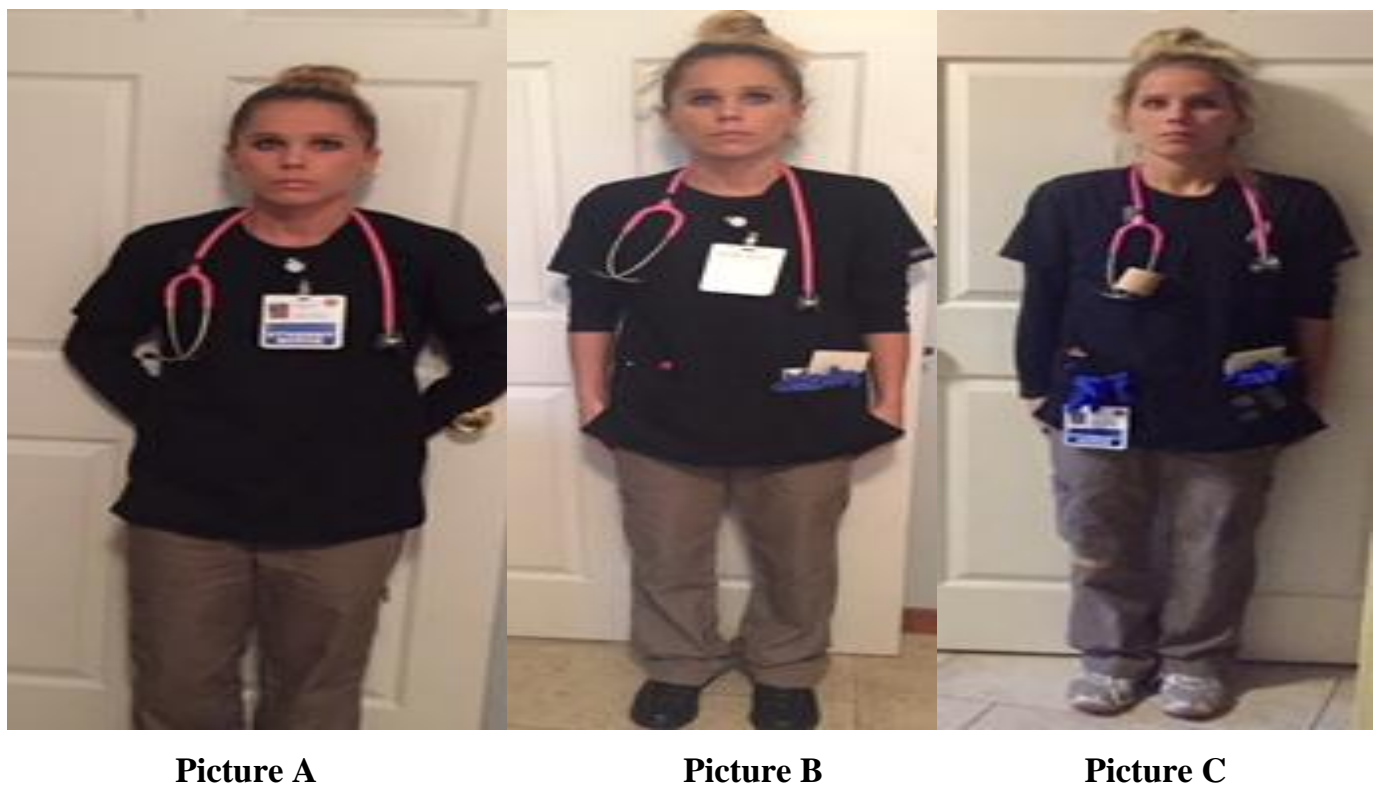

Figure 1. Image of a nurse based on time spent on personal appearance

Picture A: Sufficient Time Spent on Personal Appearance

Picture B: A Little Time Spent on Personal Appearance

Picture C: Very Little or No Time Spent on Personal Appearance

\subsection{Picture B: A Little Time Spent on Personal Appearance}

In Table 3, the statements had a mean between 2.31 and 2.81. As shown in picture B, most of the participants agreed that the image displayed preparedness $(\mathrm{M}=2.81, \mathrm{SD}=0.68)$, and professionalism $(\mathrm{M}=2.59, \mathrm{SD}=0.69)$. Other statements ranked lower, including confidence $(\mathrm{M}=2.61, \mathrm{SD}=0.69)$, trustworthiness $(\mathrm{M}=2.57, \mathrm{SD}=0.67)$, and compassion ( $M=2.42, S D$ 0.62). The lowest level of agreement was laziness $(M=2.31, S D=0.74)$. Picture $B$ was perceived as being prepared and confident. Picture B ranked lower in compassion and trustworthiness. Picture B had less attentiveness in appearance than Picture A, and the results show this connection. Picture B had a lower mean for all of the asked statements. Picture B was ranked lower than Picture A. When comparing Picture B to Picture A, the research validated the assumption in that more attentiveness spent on appearance would be perceived as more professional. Picture B demonstrated how less time spent on appearance could affect the perceived professionalism of the nurse.

Table 3. The appearance of the nurse and perceived professionalism Picture B

\begin{tabular}{lcc}
\hline Variable & M & SD \\
\hline I feel picture B shows preparedness & 2.81 & 0.68 \\
I feel picture B shows confidence & 2.61 & 0.69 \\
I feel picture B shows professionalism & 2.59 & 0.69 \\
I feel picture B shows trustworthiness & 2.57 & 0.67 \\
I feel picture B shows compassion & 2.42 & 0.62 \\
I feel picture B shows laziness & 2.31 & 0.74 \\
\hline
\end{tabular}

Note. $(N=120)$. Items were rated on a 4-point Likert-type scale ranging from 1 (Strongly Disagree) to 4 (Strongly Agree), higher means indicate a higher level of agreement

\subsection{Picture C: Very Little or No Time Spent on Personal Appearance}

In Table 4, the statements had a mean from 2.23 to 2.74 , and for picture $C$, most of the participants agreed that the image displayed preparedness $(\mathrm{M}=2.74, \mathrm{SD}=0.90)$, and professionalism $(\mathrm{M}=2.53, \mathrm{SD}=0.91)$. Other statements ranked lower, including trustworthiness $(M=2.4, S D=0.80)$, laziness $(M=2.39, S D=0.94)$. The lowest level of frequency was compassion $(\mathrm{M}=2.23, \mathrm{SD}=0.68)$. Picture $\mathrm{C}$ demonstrated that less attentiveness to 
appearance was viewed lower in professionalism. One interesting finding was that in picture C, having pockets full of equipment needed for daily job tasks were perceived as being prepared.

Table 4. The Appearance of the nurse and perceived professionalism Picture C

\begin{tabular}{llc}
\hline Variable & M & SD \\
\hline I feel picture C shows preparedness & 2.74 & 0.90 \\
I feel picture C shows professionalism & 2.53 & 0.91 \\
I feel picture C shows trustworthiness & 2.4 & 0.80 \\
I feel picture C shows laziness & 2.39 & 0.94 \\
I feel picture C shows confidence & 2.24 & 0.86 \\
I feel picture C shows compassion & 2.23 & 0.68 \\
\hline
\end{tabular}

Note. $(N=120)$. Items were rated on a 4-point Likert-type scale ranging from 1 (Strongly Disagree) to 4 (Strongly Agree), higher means indicate a higher level of agreement

\subsection{Qualitative Themes}

The qualitative question was, "Please describe in your own words how the nurse's appearances portray professionalism." The images portrayed a nurse in various states of a professional dress with the focus on the uniform. The model was not smiling in any of the pictures, maintaining a neutral facial expression that would not skew participant impressions of the images. Recurring themes included smiling and uniform colors (see Figure 2). Participates did not want a nurse wearing dark, gloomy colors. It was discovered that people want a nurse who looks approachable and friendly. The emerged themes were that they liked a nurse to be seen as a professional but also caring and trustworthy. It is perceived that participants want a well-rounded nurse. A surprising theme discovered was that some participants felt that pockets filled with supplies showed preparedness. Being prepared for daily tasks associated with being seen as providing good quality of care. The results showed the complex nature of the nursing image.

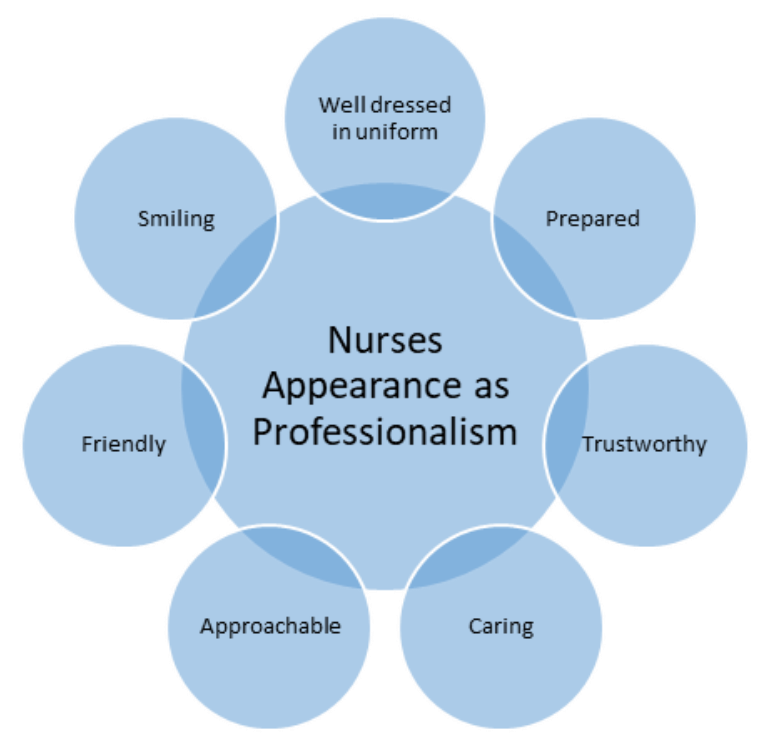

Figure 2. Qualitative themes portraying what characteristics are needed for the nurse to be perceived as exhibiting professionalism

\subsection{Summary of Results}

The research question for this study was, "How do college students perceive the professional appearance of the nurse?" The results indicated that college students felt that Picture A demonstrated a more professional and prepared nurse. After calculating the mean for all statements, Picture A showed both professionalism and preparedness with the highest mean of 3.25 ( $\mathrm{SD}=0.57)$, allowing a conclusion to be drawn that Picture A 
displays a more professional and prepared nurse. Picture A was rated lowest with trustworthy and compassion; however, the mean of $2.81(\mathrm{SD}=0.70)$ for Picture A was above the midpoint and higher than the highest mean of Pictures B and C. Picture B showed that students felt the nurse was more prepared than professional which was the same outcome for Picture C. Students see pockets full of supplies as readiness to work. The findings demonstrated the complexity of the nursing image. Laziness was identified as Picture A least lazy, Picture B moderate lazy, and Picture $\mathrm{C}$ as most lazy.

\section{Discussion of Findings}

The review of the literature indicated that professionalism was a multidimensional term. The literature review espoused that many influences could affect a person's opinion. The results indicated that Picture A exemplified the image of a professional and prepared nurse. Students ranked trustworthiness higher than confidence for this image. Of the 120 participants, only one person had not seen a nurse in uniform. Therefore, presenting a professional image presents a lasting impression for a patient, projecting competency. The theoretical framework posited that a nurse who spent more time preparing themselves for their day would be perceived as more professional. The research findings supported the theoretical framework that a person who spends more time on themselves will be portrayed as more professional. The study confirmed that the amount of time spent on appearance influenced the perceived professionalism of the nurse. The participants affirmed higher professionalism in the more attentive picture.

Upon analyzing the data for Picture B, the participants had higher agreement for preparedness. This further coincides with the literature that a nurse's image includes professionalism, confidence, preparedness, trustworthiness, and compassion. The participants want to see a nurse who is prepared for the day. The results of the survey reflected the complexity of professionalism.

The analysis of Picture $\mathrm{C}$ offered interesting insights. Preparedness was ranked higher than professionalism, with trustworthiness ranked higher than laziness. However, this image ranked higher for laziness compared to the other two pictures. This finding demonstrated how a negative image could affect professionalism. A negative image is created when a nurse wears wrinkled, dirty uniforms, has poor grooming, is unclean, has unkempt hair, and has poor posture. A negative image will decrease the nurse's credibility.

The data indicated that participants preferred a nurse who was well groomed. Someone who spends more time on appearance is perceived as more professional and competent. The data also revealed that when a nurse pays little or no attention to personal appearance, perceptions of professionalism diminished and were replaced by impressions of laziness. These findings match with previous research on professionalism. The survey disclosed that a clean and well-groomed nurse appeared professional.

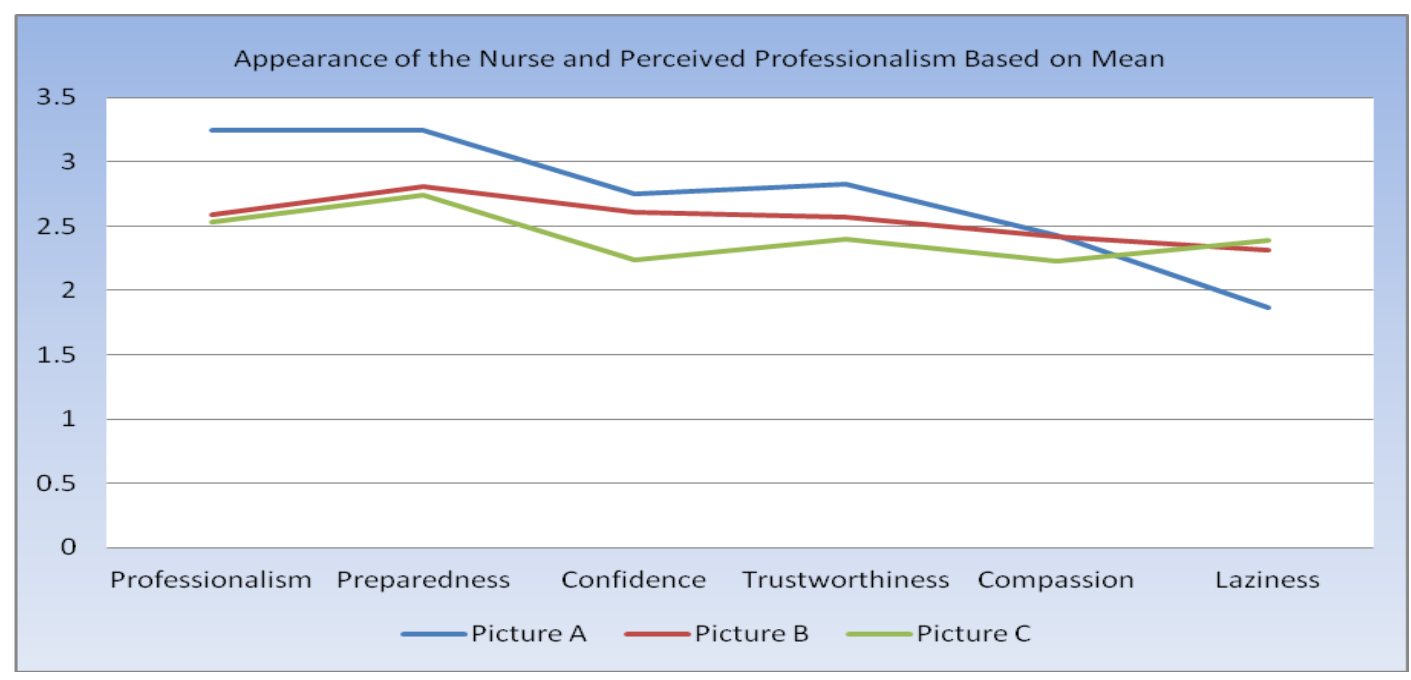

Figure 3. Comparing pictures, A, B, and C of the appearance of the nurse and perceived professionalism based on mean. Items were rated on a 4-point Likert-type scale ranging from 1 (Strongly Disagree) to 4 (Strongly Agree), higher means indicate a higher level of agreement

Note. $(\mathrm{N}=120)$ 
Figure 3 compares all three pictures in one. This figure demonstrates the complexity of professionalism. Professionalism is affected in many ways. Figure 3 shows regardless of the how the nurse looked, the participants scored them high for professionalism and low for laziness. Picture $\mathrm{C}$ also indicated lack of confidence and compassion. The least lazy was Picture A and was perceived by the participants to be well-prepared and professional.

\section{Limitations/Weakness}

A limitation of this study was the location where the survey took place because students who were at the library may have taken the study more seriously, probably leading to a better view of professionalism. The college is a private Christian based organization, which may have contributed to other possible biases. The small sample size of 120 students with the most common age range between 18-20 (61\%), and more females (59\%) than males $(41 \%)$ surveyed, limited the generalizability of findings. Regardless of limitations, valuable data were collected.

\section{Nursing Implications}

Obtaining information about how nurses are perceived in the professional world helps all nurses to be viewed as professionals. This study was important to the nursing field because nurses need to be viewed as professionals for patients to trust in their ability to care. If the public does not have trust in nurses, then health outcomes will suffer. As noted earlier, a professional image enhances credibility. Appearance and quality of care are related as shown by the research findings. People feel safer with a nurse they trust and view as professional, including appearance. Nurses should strive to be perceived as professional in every aspect.

\section{Conclusion}

In conclusion, a nurse's appearance was related to how professional others perceived them. Survey participants agreed that a well-groomed, clean, neat, and professional nurse assisting with their care is important. There is a need for further understanding of what makes a nurse professional to help improve the nursing image. Therefore, this survey shed light on how students perceived professionalism through appearance. Every aspect of care from communication to skill level is judged on the nurse's personal appearance.

\section{Recommendations}

The study was conducted to gain insight into perceptions college students had about nurse appearance and professionalism. Participant responses revealed that students prefer a nurse who values attention to appearance through personal grooming including makeup, hair, and a uniform that is neat and clean. First impressions are lasting, and it is important that student nurses get into the habit of spending time on their appearance while preparing for clinical rotations in nursing school.

The results of this survey demonstrate how nurses should portray themselves to be seen as professional. It is never too early to work on one's professional persona. Based on these findings, steps should be taken to understand the value of perceived professionalism. Recognizing that personal appearance elevates credibility and a positive image among patients should encourage the nurse to pay attention to the outward appearance that reflects professionalism.

Further research needs to be conducted in this area to compare ideas from different colleges and age groups. Gathering data from larger colleges both private and the public would help to increase the sample size and diversity. Research could be further expanded asking about the amount of jewelry, makeup, and tattoos worn by a nurse and perceived professionalism. Professionalism is a very complex issue that is always evolving.

\section{References}

Akhtar-Danesh, N., Baumann, A., Kolotylo, C., Lawlor, Y., Tompkins, C., \& Lee, R. (2013). Perceptions of professionalism among nursing faculty and nursing students. Western Journal of Nursing Research, 35(2), 248-271. https://doi.org/10.1177/0193945911408623

Clavelle, J., Goodwin, M., \& Tivis, L. (2013). Nursing professional attire: Probing patient preferences to inform implementation. The Journal of Nursing Administration, 43(3), 172-177. https://doi.org/10.1097/NNA.0b013e318283dc78

Fogle, C., \& Reams, P. (2014). Taking a uniform approach to nursing attire. Issues in Nursing, 44(6), 50-54. https://doi.org/10.1097/01.NURSE.0000444535.96822.3b

Ghadirian, F., Salsali, M., \& Cheraghi, M. (2014). Nursing professionalism: An evolutionary concept analysis. Iranian Journal of Nursing and Midwifery Research, 19(1), 1-10. Retrieved from http://ijnmr.mui.ac.ir/index.php/ijnmr/article/view/980/713 
Hartfield, L., Pearce, M., Guidice, M., Cassidy, C., Samoyan, J., \& Polomano, R. (2013). The professional appearance of registered nurses: An integrated review of peer-refereed studies. The Journal of Nursing Administration, 43(2), 108-112. https://doi.org/10.1097/NNA.0b013e31827f2260

Orem, D.E., Renpenning, K.M., \& Taylor, S.G. (2003). Self-care theory in nursing: Selected papers of Dorothea Orem. New York: Springer Publishing Company.

Porr, C., Dawe, D., Lewis, N., Meadus, R., Snow, N., \& Didham, P. (2014). Patient perception of contemporary nurse attire: A pilot study. International Journal of Nursing Practice, 20(2), 149-155. https://doi.org/10.1111/ijn.12160

Pullen, R., \& Alley, K. (2015). Does your professional image need a makeover? Nursing Made Incredibly Easy, 13(4), 22-27. https://doi.org/10.1097/01.NURSE.0000473412.68586.16

Schmidt, N.A., \& Brown, J.M. (2015). Evidence-based practice: appraisal and application of research (3rd ed., p. 358). Burlington, MA: Jones \& Bartlett Learning.

Splendore, R., Burns, H., Choby, C. (2016, April). Dress for respect: A shared governance approach. Nursing Management. https://doi.org/10.1097/01.NUMA.0000481793.90556.1b

Swan, B., \& McGinley, M. (2016). Nurse-patient communication: A catalyst for improvement. Nursing Management, 47(6), 26-28. 2016. https://doi.org/10.1097/01.NUMA.0000483129.93849.72

While, A. (2013). Attire and district nursing. British Journal of Community Nursing, 18(2), 102. Retrieved from http://connection.ebscohost.com/c/articles/85231138/whiles-words-attire-district-nursing

Wocial, L., Sego, K., Rager, C., Laubersheimer, S., \& Everett, L. (2014). Image is more than a uniform: The promise of assurance. The Journal of Nursing Administration, 44(5), 298-302. https://doi.org/10.1097/NNA.0000000000000070

\section{Copyrights}

Copyright for this article is retained by the author(s), with first publication rights granted to the journal.

This is an open-access article distributed under the terms and conditions of the Creative Commons Attribution license (http://creativecommons.org/licenses/by/4.0/). 